\title{
The Constellation-X Spectroscopy X-Ray Telescope
}

\author{
Robert Petre $^{*_{1}}$, David Content ${ }^{2}$, John Lehan ${ }^{1.3}$, Stephen O'Dell ${ }^{4}$, Scott Owens ${ }^{2}$, William Podgorsky ${ }^{5}$, \\ Jeff Stewart ${ }^{6}$, William Zhang ${ }^{1}$ \\ ${ }^{1}$ X-ray Astrophysics Branch, Code 662, NASA / GSFC, Greenbelt, MD 20771 USA \\ ${ }^{2}$ Optics Branch, Code 551, NASA / GSFC, Greenbelt, MD 20771 USA \\ Universities Space Research Associates, \\ ${ }^{4}$ Space Science Department, NASA / MSFC, Huntsville, AL 35812 USA \\ ${ }^{5}$ Smithsonian Astrophysical Observatory, 60 Garden Street, Cambridge, MA 02138 USA \\ ${ }^{6}$ Mechanical Systems Branch, Code 543, NASA / GSFC, Greenbelt, MD 20771 USA
}

\begin{abstract}
The status of technology development for the Constellation-X Spectroscopy X-ray Telescope (SXT) mirror is presented. The SXT mirror combines a large $(1.6 \mathrm{~m})$ aperture with modest $(12 \mathrm{arcsec}$ half power diameter) angular resolution and low mass (750 kg). The overall collecting area, larger than 9,600 square $\mathrm{cm}$ at $0.25 \mathrm{keV}$, requires high throughput, and thus nesting of a substantial number of thin reflectors. A phased development program is underway to develop reflectors, mounting and alignment approaches, and metrology techniques for components and the mirror has a whole. The latest results in all these areas are summarized, along with an overview of results of optical testing of reflector performance.
\end{abstract}

Keywords: X-ray mirrors, Constellation-X

\section{INTRODUCTION}

The Spectroscopy X-ray Telescope (SXT) is the heart of Constellation-X, NASA's next major X-ray observatory. The SXT consists of a large, high throughput mirror, a reflection grating that intercepts half the converging mirror beam and diverts it to CCD, and a calorimeter that collects the radiation not diverted by the grating. The SXT mirror represents the enabling technology for the mission, and offers a significant technological challenge. The challenge arises from the combination of required properties - low mass, high throughput over a broad band, and moderate angular resolution. As the Constellation- $\mathrm{X}$ mission comprises four identical observatories, an additional challenge is mass production of optical components.

The critical performance requirements for the SXT mirror are compiled in Table 1

\section{SXT MIRROR DESIGN}

Our implementation choice for the SXT mirror has its heritage in the thin-foil, high-throughput, conical approximation mirrors developed at the GSFC, and implemented on the Broad Band X-ray Telescope (BBXRT), ASCA, and Astro-E (ref). These mirrors combine high-throughput, low mass, and modest angular resolution with a modular design that has lent itself to mass production. Given the substantial diameter difference between conical mirrors (largest diameter 40 $\mathrm{cm}$ ) and the SXT mirror (diameter $1.6 \mathrm{~m}$ ) and the higher required angular resolution, a substantial modification of the conical mirror production approach is needed for the SXT. While the basic elements of the conical mirror are preserved in the SXT mirror design (modular housings, segmented reflectors, mass production, epoxy replication of the reflecting surfaces), every component of the mirror has undergone significant evolution. A schematic of the current SXT mirror design is shown in figure 1. It incorporates the following attributes:

- Thin $(400 \mu \mathrm{m})$ glass reflector substrates, precisely shaped to Wolter I figure via heat forming, and coated with a

robert.petre-1@nasa.gov; phone 301-286-3844; fax 301-286-0677 
thin $(<10 \mu \mathrm{m})$ layer of epoxy to remove residual mid-frequency ripples.

- Housings constructed out of a Ti alloy with an identical coefficient of thermal expansion to that of the glass.

- An alignment approach that allows reflectors to be aligned individually, via a closed loop system incorporating actuators and optical measurements.

\begin{tabular}{|c|c|c|}
\hline \multicolumn{3}{|c|}{$\begin{array}{c}\text { Table } 1 \\
\text { SXT mirror requirements }\end{array}$} \\
\hline \multicolumn{3}{|c|}{ Performance requirements } \\
\hline Bandpass & $0.25-10.0 \mathrm{keV}$ & \\
\hline \multicolumn{3}{|c|}{ Effective area (per mirror) } \\
\hline$@ 0.25 \mathrm{keV}$ & $8,825 \mathrm{~cm}^{2}$ & $33,000 \mathrm{~cm}^{2} @ 1.25 \mathrm{keV}$ and $6,900 \mathrm{~cm}^{2} @ \mathrm{t} 6.0 \mathrm{keV}$ for mission. \\
\hline$@ 1.25 \mathrm{keV}$ & $8,420 \mathrm{~cm}^{2}$ & Allows for losses due to grating and detector inefficiency. \\
\hline$@ 6.0 \mathrm{keV}$ & $1,720 \mathrm{~cm}^{2}$ & \\
\hline Angular resolution & 12.5 arc seconds HPD & Consistent with observatory HPD of 15 arc seconds. \\
\hline & 4 arc seconds HPD goal & Consistent with observatory HPD goal of 5 arc seconds \\
\hline Field of view & 2.5 arc minutes & $\begin{array}{l}\text { Defined by detector field of view } \\
>90 \% \text { of on-axis effective area at } 1.25 \mathrm{keV} \text { across field of view }\end{array}$ \\
\hline \multicolumn{3}{|l|}{ Derived requirements } \\
\hline Diameter & $1.6 \mathrm{~m}$ & To meet effective area requirement with four mirrors. \\
\hline Focal length & $10 \mathrm{~m}$ & To assure required area at $6 \mathrm{keV}$ using $1.6 \mathrm{~m}$ diameter mirrors. \\
\hline Reflector axial length & $>20 \mathrm{~cm}$ & To fit within envelope and meet fabrication considerations. \\
\hline Operating temperature & $20 \pm 1 \mathrm{C}$ & Keeps thermal distortions from driving HPD beyond 12.5 arc sec. \\
\hline Mass & $650 \mathrm{~kg}$ & Current engineering estimate. \\
\hline
\end{tabular}

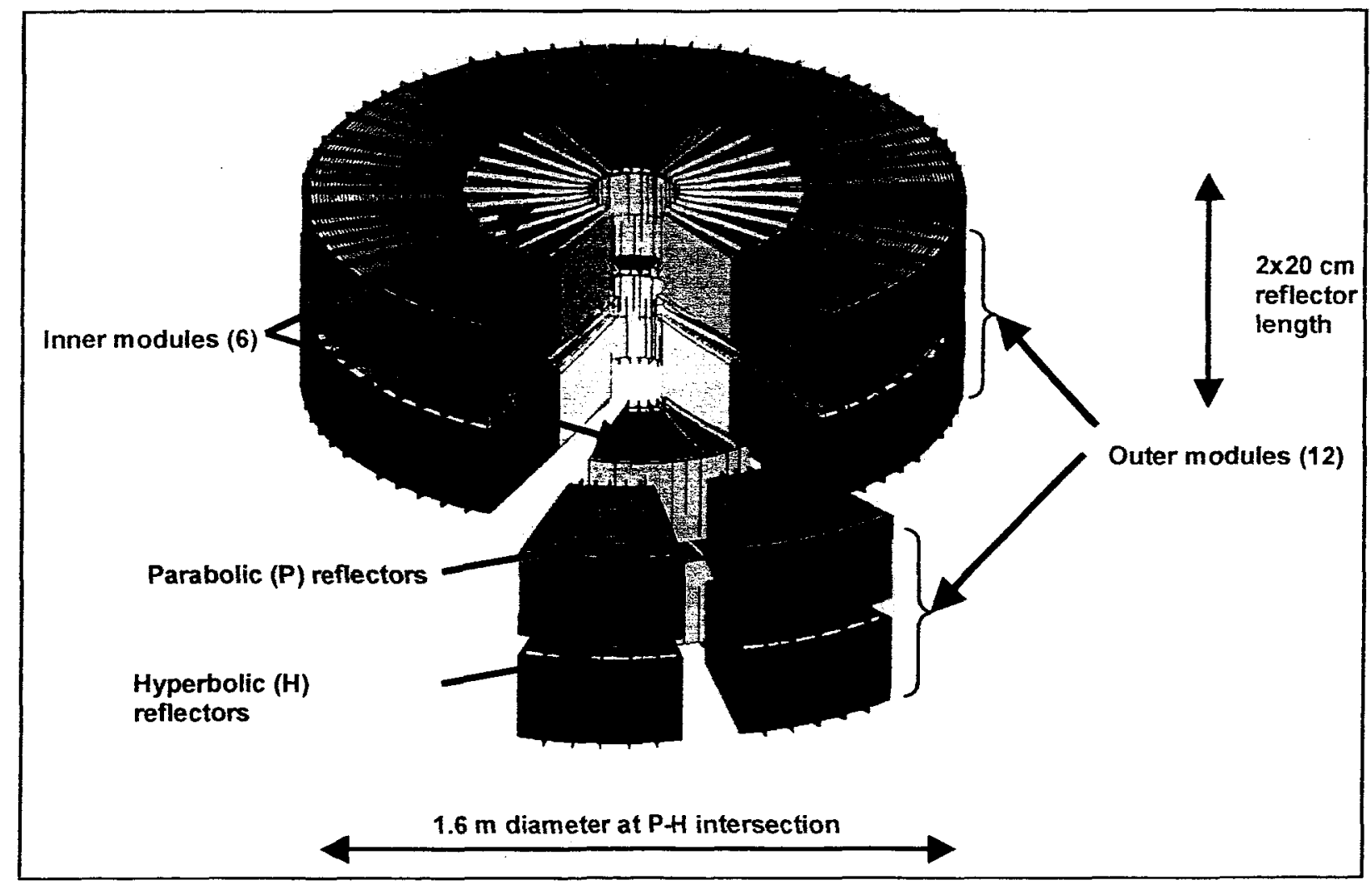

Figure 2: Schematic of the Constellation-X SXT mirror. 
The mirror design is highly modular, with twelve outer modules (each subtending a 30 degree arc) and six inner modules (subtending a 60 degree arc). The boundary between the inner and outer modules is as approximately a radius of $0.4 \mathrm{~m}$. In that way the largest reflectors in the inner and outer modules have the same size. The highly modular approach facilitates mass production of reflectors. The key features of the mirror design are captured in Table 2.

Table 2

SXT mirror baseline design parameters

Design

Reflector substrate material

Reflecting surface fabrication

$\mathrm{X}$-ray reflecting surface

Number of nested reflectors

Total number of reflectors

Reflector axial length

Number of modules

Module housing composition

Largest reflector surface area

Substrate density

Reflector thickness

Reflector microroughness

\author{
Segmented Wolter I \\ Thermally formed glass \\ Epoxy replication \\ Gold \\ 140 (inner module); 90 (outer module) \\ 3,840 \\ $20 \mathrm{~cm}$ \\ 6 (inner); 12 (outer) \\ Ti alloy, CTE-matched to substrate \\ $0.16 \mathrm{~m}^{2}$ \\ $2.4 \mathrm{~g} \mathrm{~cm}^{-3}$ \\ $0.4 \mathrm{~mm}$ \\ $0.4 \mathrm{~nm}$ RMS
}

Technology development for the SXT mirror has been underway for several years, and has been following a welldefined roadmap in which progressively larger and more flight-like units are constructed. ${ }^{2}$ Recent funding cutbacks within NASA have forced the technology development to proceed more slowly, however. Prior to the reduction of funding, the SXT mirror program had reached a point when a major facility investment would be required to produce the next, considerably larger unit (incorporating $1.6 \mathrm{~m}$ reflector segments, the largest ones in the baseline design). Instead, technology development has continued to concentrate on components of a small-scale (50 cm diameter) mirror module. All technology development work is expected to be applicable to a full-sized mirror system, largely as a result of the system modularity.

In the sections below, we discuss the development carried out by the SXT mirror team over the past year.

\section{REFLECTORS}

The reflector surface quality is the largest term in the SXT mirror angular resolution error budget. To meet a system level angular resolution requirement of 15 arc seconds half power diameter (HPD), the mirror must contribute no more than 12 arc seconds. The error budget share allotted to the reflector surfaces is $\sim 10$ arc seconds. Thus each reflector must contribute less than 7 arc seconds to the image HPD. If the angular resolution goal of 5 arc seconds HPD is to be met, each reflector must contribute less than 2.5 arc seconds to the image HPD, Since the reflectors are the largest source of blur, fabricating them accurately is the key to meeting or exceeding the angular resolution requirement. The SXT mirror team has therefore concentrated a substantial effort to improving the quality of the replicated reflectors and the underlying substrates. ${ }^{3}$

The SXT team recognized over a year ago that its approach to reflector fabrication needed modification. ${ }^{2.4}$ Originally we intended to use the heat forming to shape the substrates to the approximate shape (within a few microns), apply the final axially-curved surface via epoxy replication, and correct for low order azimuthal errors during alignment. This approach required a relatively thick $(\sim 40 \mu \mathrm{m})$ epoxy layer, whose shrinkage stressed the glass, causing deformation. Reducing the epoxy thickness required several major changes. First, the local surface irregularities in the formed substrate had to be reduced. As our rule of thumb for epoxy thickness is that smoothing out features of a particular height requires an epoxy thickness ten times the feature height, making the epoxy layer thinner imposed very strong constraints on surface irregularities. The irregularities arise from two sources: dust trapped between the forming mandrel and the substrate, and graininess of the release layer on the forming mandrel. We invested substantial effort in improving the cleanliness of the glass-forming laboratory, introducing laminar flow clean tents around the oven and substrate handling area. We 
have also investigated candidate release layer materials, to determine which provides the right combination of smoothness, low adhesion, and lubricity. The result of these improvements is that formed substrates now faithfully reproduce the mandrel surface on spatial scales smaller than $1 \mathrm{~cm}$. Moreover, the typical small surface feature amplitude is now less than $0.5 \mu \mathrm{m}$, allowing a substantial reduction of epoxy thickness.

Two major changes have been introduced for controlling the overall figure of the substrates. A detailed finite element analysis was performed of the forming process, identifying where and when thermal gradients develop, and the consequent non-uniformities introduced into the substrate figure. From this analysis, we identified and performed several modifications forming oven and to the heating and cooling profiles. The second change is to use forming mandrels figured to the nominal Wolter I surface. This allows the application of a uniform epoxy layer, whereas the use of conical forming mandrels required reliance on the epoxy layer to form and hold the surface figure. The major change is less the actual figure as much as it is the recognition that long wavelength surface deviations must be avoided. Figuring that mandrel surfaces to a Wolter geometry is no more difficult than figuring them to conical geometry. GSFC's Optics Branch has figured the surface of a pair of $50 \mathrm{~cm}$ diameter, fused Si forming mandrels. Each has a geometric HPD of less than 4 arc seconds, so their optical quality is approximately as high as the superpolished $50 \mathrm{~cm}$ $\mathrm{Ni}$-coated $\mathrm{Al}$ mandrel we use for replication.

The net result of these changes is shown in Figures 2,3 and 4. Figure 2 shows overlaid surface profile curves at three aximuthal locations for five different substrates formed consecutively. The surfaces are identical at the submicron level, demonstrating the quality of the substrates and the stability of the forming process. Figure 3 shows surface profiles at five azimuthal locations for the first substrate formed using a Wolter mandrel. The surface is uniform, has the correct sense of axial curvature, and shows no low order distortions. Figure 4 show's a "modified" power spectral density (PSD) plot of a recent reflector. This particular reflector was formed using all the process changes, but was formed against a mandrel that has not been figured. The measured PSD is compared against the profile required for a reflector to meet its share of the system angular resolution error budget. The plot shows that over most spatial frequencies, the SXT requirement is met or exceeded.

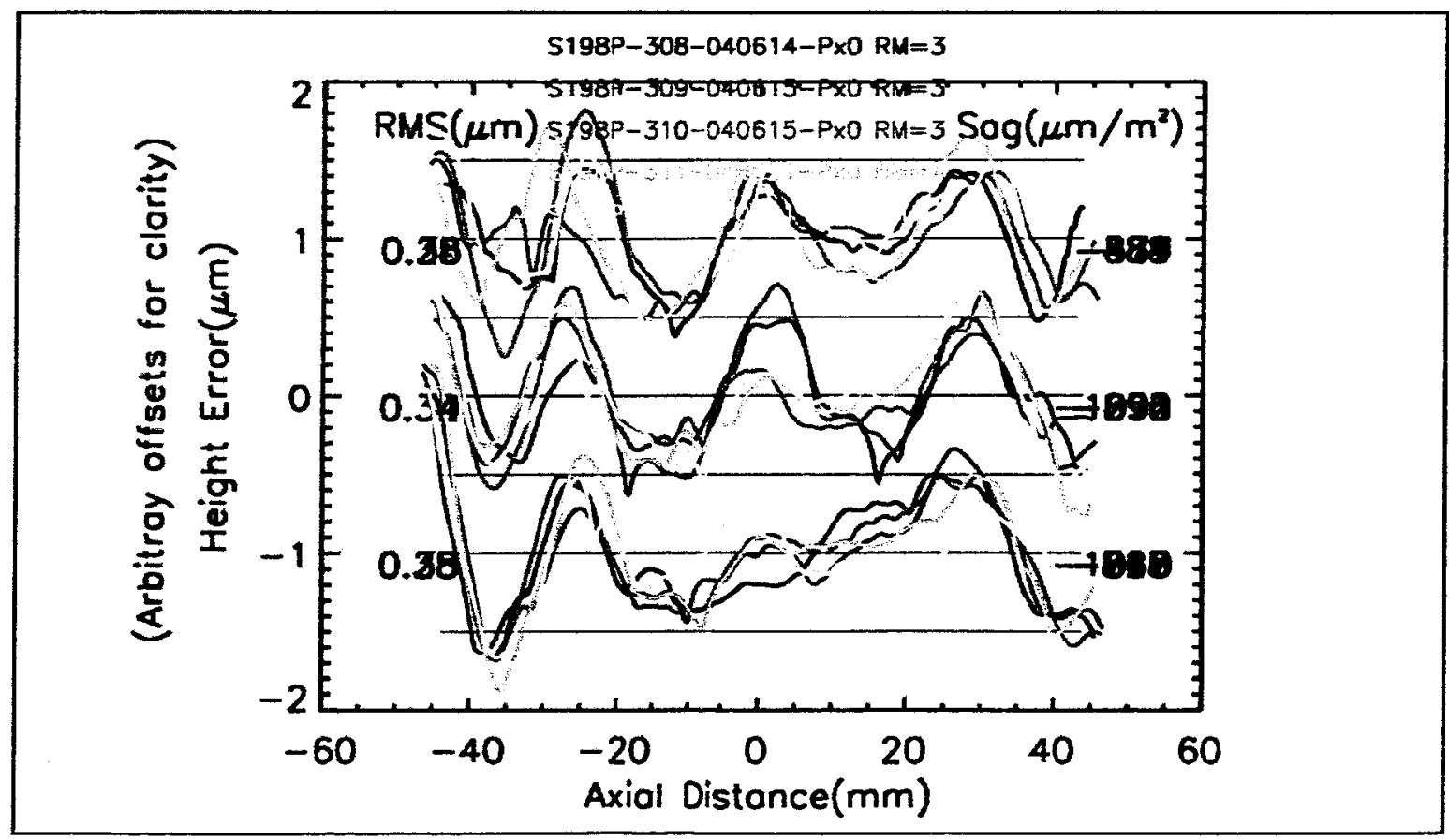

Figure 2: Axial profiles at three azimuthal positions of five reflector substrates, formed consecutively. The profiles follow each other identically, indicating that the residual structure is intrinsic to the forming mandrel and not the formed substrates. Note that the vertical scale is microns. 


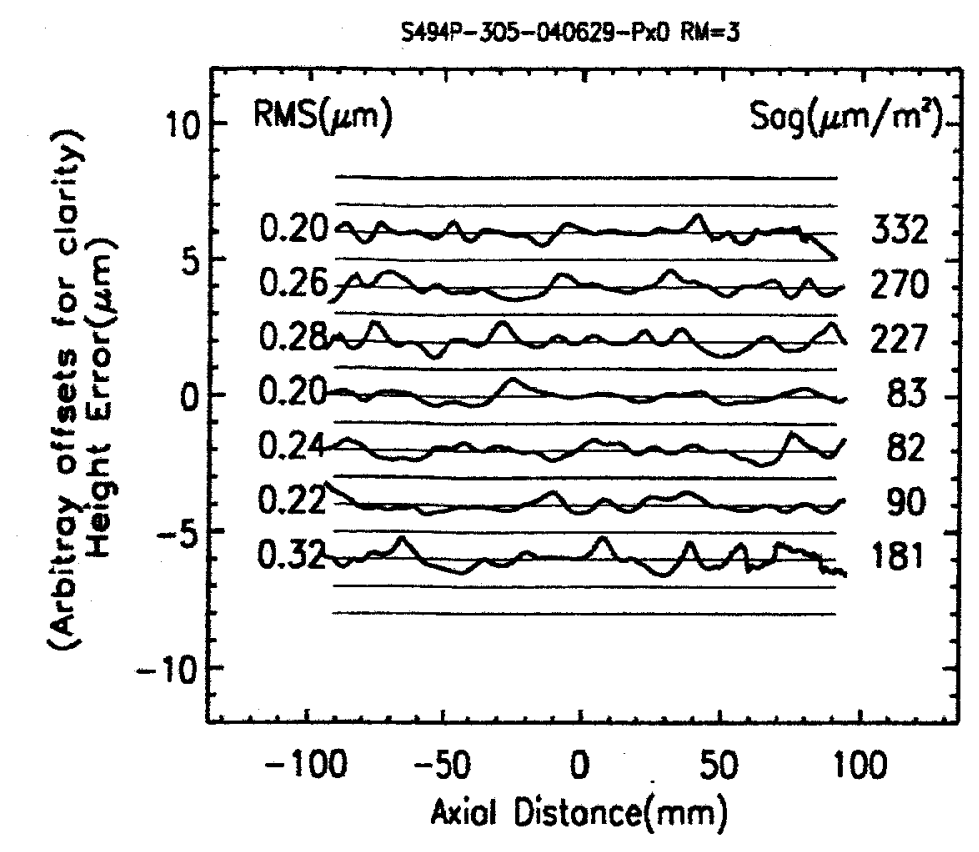

Figure 3: Axial scans at five azimuths of the first substrate formed on a certified Wolter-I mandrel. The left hand scale is the rms deviation from the nominal surface in $\mu \mathrm{m}$; the right hand scale is the depth of the curvature. The sag for a perfect reflector is approximately 150 in the units displayed. The most likely reason for the incorrect sag is gravity/metrology distortion rather than intrinsic forming error.

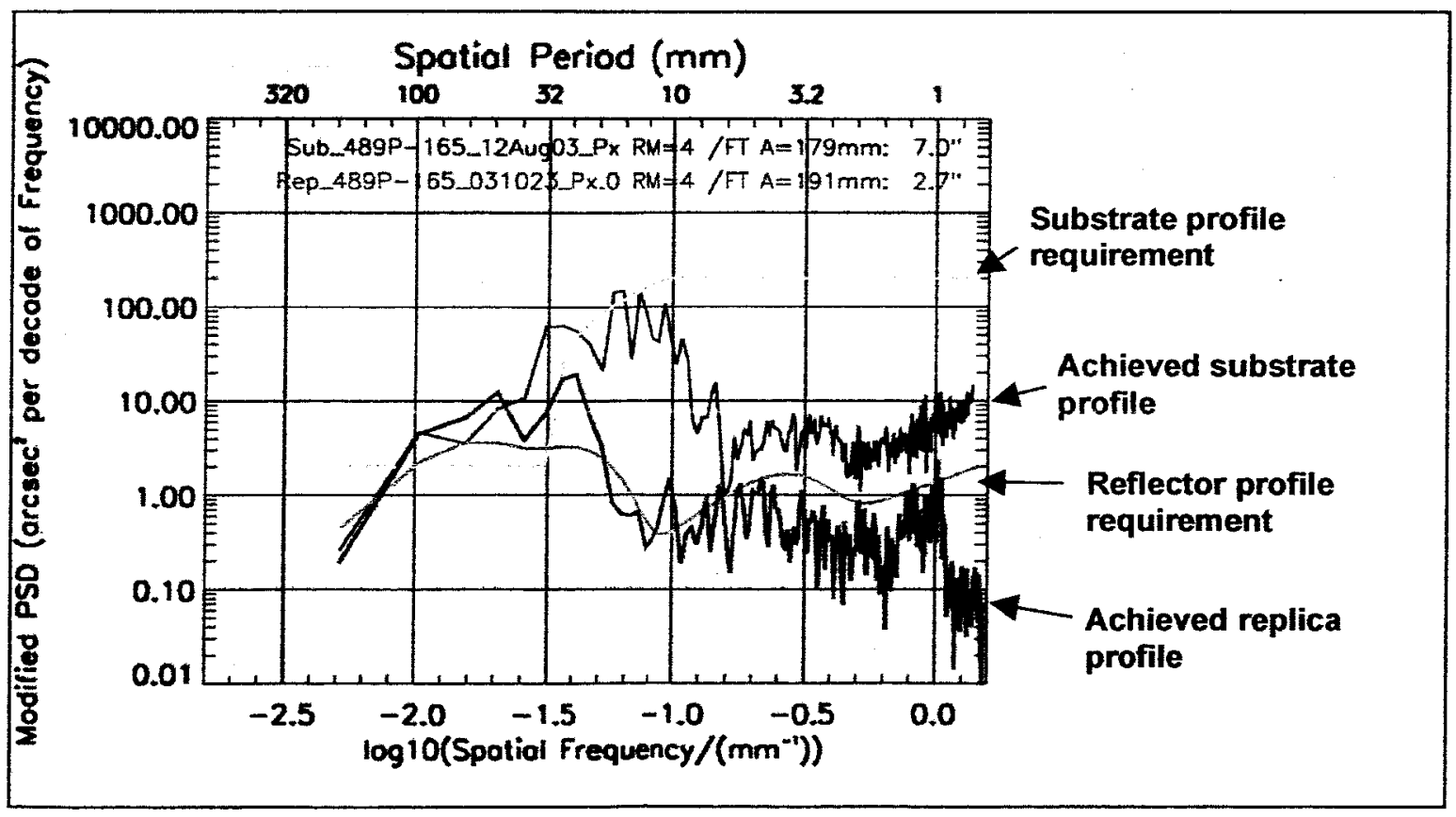

Figure 4: A comparison of the modified power spectral density (PSD) plot of a recent reflector, before and after reflection, with that needed to meet the angular resolution error budget allotment. The top smooth curve shows the PSD requirement for the bare substrate; the second curve shows the measured PSD of the substrate; the third curve shows the required PSD for a finished reflector; and the bottom curve shows the measured PSD. At nearly all spatial frequencies, the PSD requirement has been met or exceeded. 


\section{REFLECTOR METROLOGY}

Measuring the surface properties of reflectors and substrates has proven to be as challenging as fabrication. This is easy to understand. As the surface features on a substrate are a fraction of a micron in amplitude, metrology must be accurate to tens of nanometers. The thin glass substrate is easily distorted; it is extremely challenging to develop a mount that presents them to a metrology instrument in their "natural" (undistorted) state. After considerable engineering and numerous models, we have finally developed a substrate mount that affords reasonably accurate (and repeatable) surface metrology. As shown in Figure 5, the mount holds a substrate at three points, applying minimum stress. A more detailed description can be found in reference 5 .

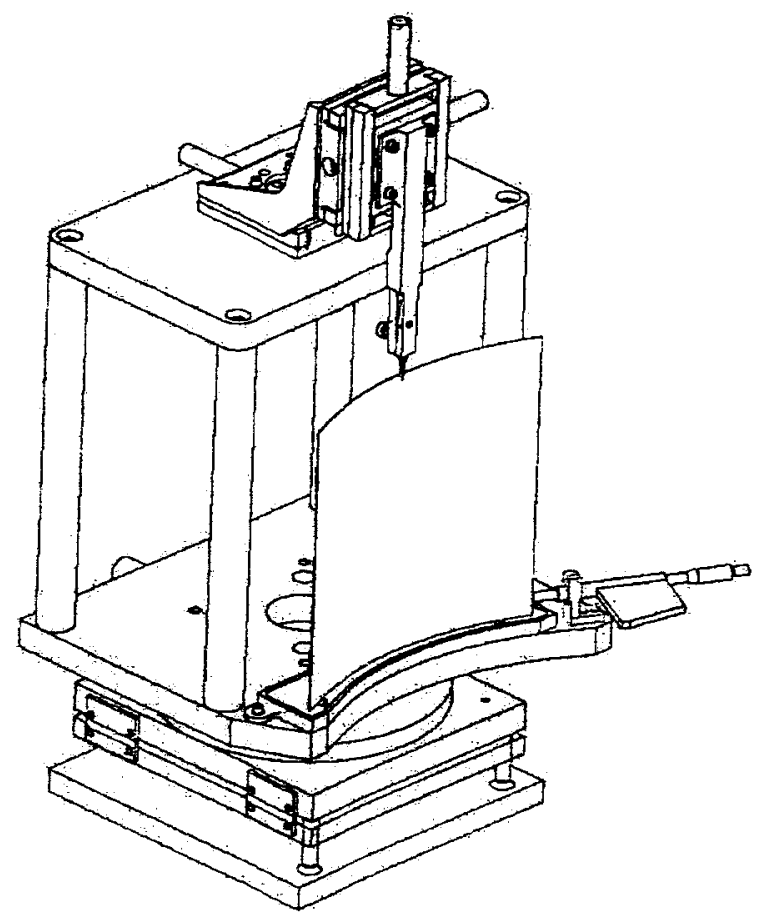

Figure 5: A minimum stress metrology mount for substrates and reflectors. A sample is held at three points, one at the top and two at the bottom. The bottom mount points are located in a well that can be filled with a viscous fluid to reduce the mounting stress.

Until recently, most substrate and reflector surface metrology relied on 1-D axial scans at a discrete set of azimuthal positions (typically five). Uncertainty is added to the metrology by the axial registration accuracy: it is extremely difficult to repeat a scan at the identical azimuth on multiple reflectors (some of the reflector-to-reflector variations in Figure 2 could be due to registration). Moreover, a small set of 1-D scans does not fully reveal the surface topology. Clearly a 2-D surface mapping approach is strongly preferred. This is another challenge as profilometry is extremely time consuming, and ordinary interferometric techniques are not applicable to the extremely aspheric mandrel and substrate surfaces. Very early on in the SXT mirror development program, we investigated the suitability of a Computer Generated Hologram $(\mathrm{GCH})$ to provide a surface map. We initially rejected their use, as the surfaces of the mandrels and reflectors at that time were too poor to attain useful data. Recently, we have determined that CGH measurements are attainable for the current generation of mandrels, substrates and reflectors. The CGH approach is shown schematically in Figure 6. An interferogram of a mandrel is shown in Figure 7. Now that the feasibility of this approach has been established, we are attempting to find ways to map an entire $20 \mathrm{~cm} \mathrm{x} 30 \mathrm{~cm}$ substrate. Ideally, this is done using a $\mathrm{CGH}$ that covers the surface in a single exposure, but such devices are not readily available commercially. While we seek source for such devices, we are in the meantime developing "stitching" algorithms that allow us to reconstruct a full surface interferogram from a set of partial surface interferograms. This turned out to be nontrivial, as the various overlapping partial surface interferograms need to be matched not only in $x$ and $y$, but in intensity, and have nonlinear 
spatial distortions removed. Figure 8 shows a recent successful stitching of interferograms from the mandrel used to create figure 7. More detail about this approach is presented in reference 5 .

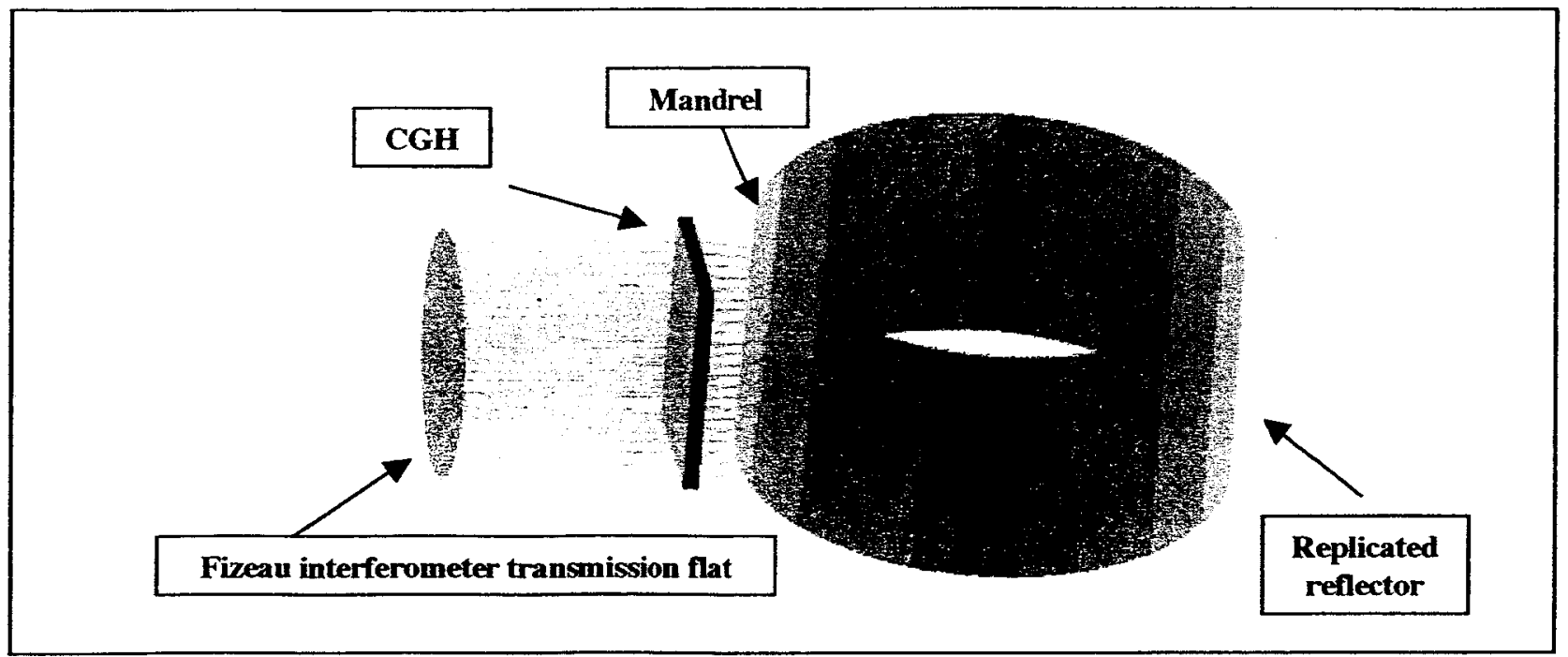

Figure 7: The CGH concept: Null testing of either a conical mandrel (first intersection) or replicated reflector ( $2^{\text {nd }}$ intersection) can be performed. The leftmost surface is the transmission flat of the Fizeau interferometer.

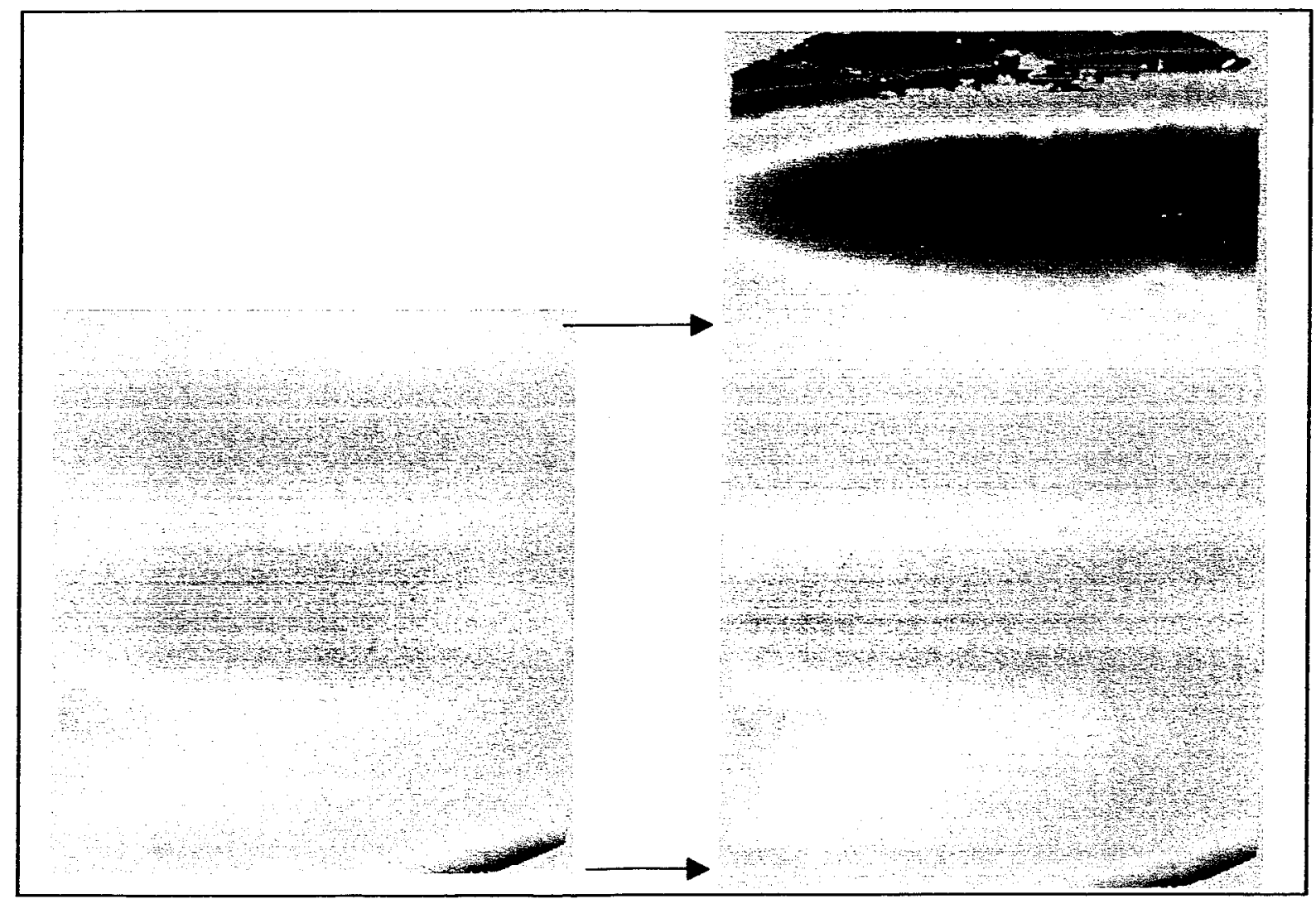

Figure 8: (left) Mandrel with nominal match between the CGH null and the test part. (right) Stitched interferogram of the mandrel shown at left. The portion shown in (a) is at the bottom $2 / 3$ of stitched result. 


\section{REFLECTOR ALIGNMENT}

The overall approach to SXT mirror alignment remains the same: alignment of each reflector or reflector pair within the flight housing. Gang alignment, however attractive, is impractical unless reflectors can be produced that conform to the nominal optical prescription to sub-micron accuracy; there are too many uncertainties in the forming and replication to guarantee this. Previous work using the Optical Alignment Pathfinders ( 1 and 2 ) has established the required number of

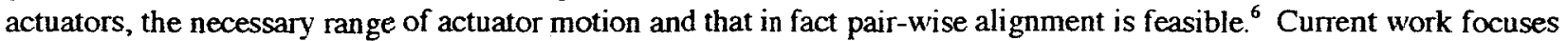
on developing an approach that is scalable to the hundreds of reflectors per housing, and possible to automate.

In principle, automation is straightforward. Alignment is carried out using the Centroid Detector Assembly (CDA) originally used for the AXAF (Chandra) mirror, ${ }^{7}$ and modified for the segmented SXT mirror. ${ }^{6}$ The alignment configuration is shown schematically in Figure 9. The CDA can detect sub-arc-second changes in the signal produced by a reflector or reflector pair, well below the sensitivity needed for the SXT. Misalignments and distortions produce recognizable patterns in the CDA output. A small movement of an actuator produces a predictable change in the return pattern to the CDA. Thus, combining the actuator motion with the CDA output into a closed loop system should produce an adequately aligned module. Experiments are underway to test this approach.

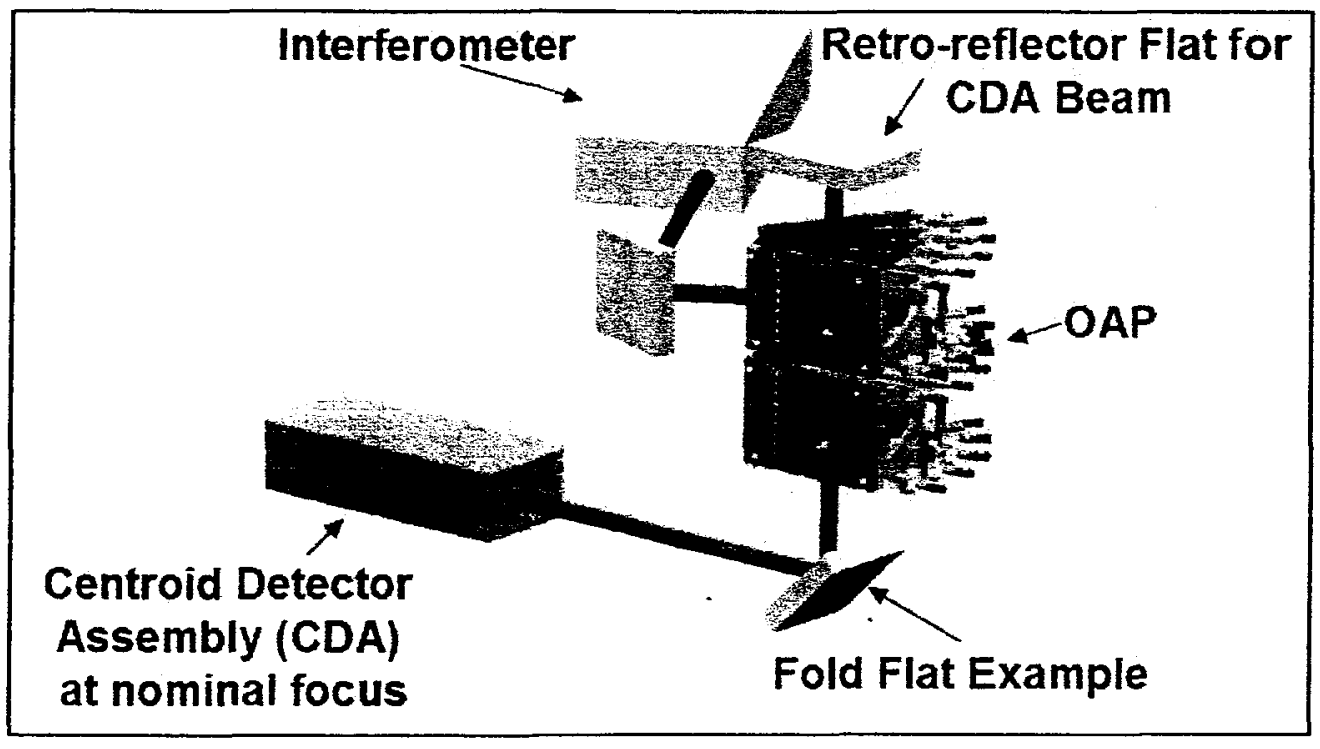

Figure 9: Schematic of the OAP alignment configuration. The CDA is used to bring rays reflecting off all portions of a reflector pair to a common focus. The interferometer is used to measure the axial curvature of the reflectors as they are manipulated within the OAP.

We are also investigating a new approach for aligning and mounting reflectors. The current approach incorporates a removable alignment system. Actuators are attached to one reflector pair at a time. The pair is aligned, the reflectors bonded to a set of struts, and the actuators are removed and attached to the next pair. This procedure is time consuming, and adjusting a reflector pair, once aligned and bonded, will be difficuit, if possible at all. A scheme under study involves the use of piezoelectric crystals as combined alignment and structural elements. This is shown schematically in Figure 10. Each reflector is mounted in the housing using five pairs (top and bottom) of bidirectional piezoelectric crystals. Thus each reflector has its own alignment mechanism built in to the housing. Once a housing is filled with reflectors, alignment is performed on each reflector by applying voltage to the crystals. Once aligned, the reflectors are bonded in place (with voltage applied). Once they are bonded, the voltage is removed, and the crystals become an integral part of the reflector support. This approach has numerous advantages. It is simpler mechanically, obviating the need for additional alignment structure. It minimizes the contact with reflectors, reducing the risk of damage. It accommodates simultaneous alignment of multiple reflector pairs. A test fixture incorporating one reflector pair is under development. 


\section{X-RAY PERFORMANCE TESTING}

The Stray Light Facility, a $100 \mathrm{~m} \mathrm{X-ray} \mathrm{test} \mathrm{facility} \mathrm{at} \mathrm{Marshall} \mathrm{Space} \mathrm{Flight} \mathrm{Center,} \mathrm{has} \mathrm{been} \mathrm{outfitted} \mathrm{for} \mathrm{performance}$ testing of SXT mirrors. ${ }^{8}$ A custom, massive $(800 \mathrm{~kg}$ ) 6 -degree-of-freedom optical table has been constructed especially for SXT mirror tests. For tests involving small numbers of $50 \mathrm{~cm}$ mirror pairs (mounted in the OAP2 frame), a special thermostatically controlled housing has been constructed to ensure that the reflectors are tested at the temperature at which they were aligned. A performance test "dry run" was carried out in late 2003 to verify the operation of the test apparatus and procedures. A pair of substrates were mounted in the OAP2 housing. This unit was installed and aligned optically in the beam. The nominal operation of the thermal housing and 6-degree-of-freedom optical table was verified The facility is therefore ready for X-ray performance testing of SXT reflector pairs. The first performance test will be scheduled once reflectors meeting the SXT mirror figure requirement have been produced and aligned.

\section{NEAR TERM PLANS}

Over the next year, the SXT team will continue to concentrate on the development of components of a $50 \mathrm{~cm}$ diameter demonstration unit. Our specific goals are:

- Produce $50 \mathrm{~cm}$ reflectors that meet or exceed performance requirement. This will be accomplished by incorporating into the substrate forming process several improvements, including new, figured forming mandrels, a new temperature cycle that minimizes thermal gradients in the mandrel, and a new forming mandrel release layer coating process. We plan to test a reflector pair in X-rays at the MSFC Stray Light Facility as soon as possible.

- Continue developing metrology for supporting substrate and reflector development. In particular we hope to refine the new low stress substrate metrology mount, and incorporate CGH mapping of full substrates into our metrology regimen.

- Implement a closed loop, automated alignment scheme. A prototype of this scheme is being developed, incorporating the OAP and the CDA. If a prototype scheme is developed soon, we will test it using the first set of high quality $50 \mathrm{~cm}$ reflectors.

\section{SUMMARY}

Recent SXT technology development has been hampered by funding reduction, but nevertheless has moved steadily toward meeting the angular resolution requirement, and developing supporting metrology and an alignment scheme that will be practical for a flight mirror. We anticipate soon having $50 \mathrm{~cm}$ reflectors which, when properly aligned, will provide an image with an HPD meeting the 12 arc second SXT mirror requirement. We anticipate carrying out an X-ray performance demonstration of such a pair within a year. In the meantime, we are investigating what process improvements will be necessary to approach the 5 arc second HPD system goal. At the same time, the SXT team is supporting the ongoing reconfiguration studies of Constellation-X; the modular SXT mirror design makes the anticipated changes straightforward to evaluate and no more of a challenge to implement than the current configuration.

\section{ACKNOWLEDGEMENTS}

Numerous talented and dedicated individuals from the SXT mirror team member institutions have contributed to the progress toward the SXT mirror goals. Special mention must be given to Ms. Diep Nguyen, the SXT mirror technology development manager. The SXT mirror is a collaboration led by NASA / GSFC, with critical contributions from SAO, NASA / MSFC, and MIT.

\section{REFERENCES}

1. Y. Soong, K.-W. Chan, P. Serlemitsos, J. Lehan, \& T. Okajima, Status of segmented thin-foil X-ray telescopes, Proc. SPIE, 5488-48, 2004.

2. R. Petre, et al., Recent progress on the Constellation-X spectroscopy X-ray telescope (SXT), Proc. SPIE, 5168, 196, 2004.

3. W. W. Zhang, Development of Lightweight Mirrors for the Constellation-X Observatory, Proc. SPIE, 5488-41, 2004.

4. W. W. Zhang et al., Development of mirror segments for the Constellation-X mission, Proc. SPIE, 5168, 168, 
2004.

5. D. A. Content et al., Optical Metrology for the Segmented Optics on the Constellation-X Spectroscopy X-ray Telescope, Proc. SPIE, 5488-78, 2004.

6. S. M. Owens et al., Constellation-X SXT optical alignment Pathfinder 2: design, implementation, and alignment, Proc. SPIE, 5168, 239, 2004.

7. P. E. Glenn, Centroid detector assembly for the AXAF-I alignment test system, Proc. SPIE, 2515, 3521995.

8. S. L. O'Dell et al., X-ray testing Constellation-X optics at MSFC's 100-m facility, SPIE Proc. 5168, 306, 2004. 